\title{
Dirk Greimann* \\ Ontological Indifference of Theories and Semantic Primacy of Sentences
}

https://doi.org/10.1515/krt-2021-0014

Published online July 9, 2021

\begin{abstract}
In his late philosophy, Quine generalized the structuralist view in the philosophy of mathematics that mathematical theories are indifferent to the ontology we choose for them. According to his 'global structuralism', the choice of objects does not matter to any scientific theory. In the literature, this doctrine is mainly understood as an epistemological thesis claiming that the empirical evidence for a theory does not depend on the choice of its objects. The present paper proposes a new interpretation suggested by Quine's recently published Kant Lectures from 1980 according to which his global structuralism is a semantic thesis that belongs to his theory of ontological reduction. It claims that a theory can always be reformulated in such a way that its truth does not presuppose the existence of the original objects, but only of some objects that can be considered as their proxies. Quine derives this claim from the principle of the semantic primacy of sentences, which is supposed to license the ontological reductions he uses to establish his global structuralism. It is argued that these reductions do not actually work because they do not account for some hidden ontological commitments that are not detected by his criterion of ontological commitment.
\end{abstract}

Keywords: Quine, structuralism, ontological reduction, context principle

\section{Introduction}

Quine's ontological development divides into the following stages. ${ }^{1}$ In his early article 'On What There is' (1948), he defended his much-discussed quantificational criterion of ontological commitment according to which a theory is committed to acknowledge the entities to which its bound variables refer. Many years later, in

1 The research presented in this article has been supported with a grant from CNPq, for which I am very grateful.

*Corresponding author: Dirk Greimann, Departamento de Filosofia, Universidade Federal Fluminense, Campus do Gragoatá, Bloco 0, sala 332, Niterói 24210-350, Brazil, E-mail: dirk.greimann@gmail.com 
'Ontological Reduction and the World of Numbers' (1964), he outlined his theory of ontological reduction. It is inspired by the structuralist view in mathematics that there are different reductive definitions of number that are equally satisfactory, although they are incompatible with each other. From this he concluded that isomorphism of extensions is both a necessary and a sufficient condition for the material adequacy of a reductive definition (see Quine 1964, p. 214). To make this more precise, he introduced the notion of a proxy function. A proxy function maps the original objects of a theory one-to-one to other objects that are supposed to supplant the original ones. It allows us to reinterpret the terms of the theory in a systematic way such that they no longer refer to the original objects, but to the proxies.

In 'Ontological Relativity' (1968), he revised his theory of ontological commitment in a fundamental way that is designed to do justice to the referential indeterminacy of terms. He used the reinterpretation of terms by means of proxy functions to show that there is no objective difference between the specification of the objects presupposed by a theory and their ontological reduction to other objects (Quine 1968a, pp. 43, 55, 61). To take this into account, he adopted a relativistic theory of ontological commitment according to which the ontological commitments of a theory depend on its translation.

Finally, in 'Things and Their Place in Theories' (1981), he used the reinterpretation of terms by means of proxy functions to establish his global structuralism, which claims that the choice of objects does not matter to any scientific theory (Quine 1981b, p. 20). In Pursuit of Truth (1990), he formulated this claim as the thesis of the 'indifference of ontology' (Quine 1990a, pp. 31-33). ${ }^{2}$ It stresses the deflationist consequence of global structuralism that our ontological choices matter less to science than we commonly thought. The proxy-function argument 'defuses' ontology to a certain extent. ${ }^{3}$

Quine reaffirmed his structuralism also in his later writings 'Structure and Nature' (1992, p. 8) and 'Naturalism; or Living Within One’s Means' (1995b, p. 259). It is his final word about ontology. These writings, however, do not clearly explain what exactly the point of his global structuralism is. Quine always stresses that the choice of objects does not matter to the empirical evidence for a theory. ${ }^{4}$ This epistemological thesis must certainly be regarded as a central doctrine of his global structuralism. ${ }^{5}$ Strictly speaking, it claims that the choice of objects for a theory is

2 The formulation of this thesis as 'structuralism' is not used in this book.

3 See Quine 1990a, p. 33.

4 See Quine 1981b, p. 21, 1983, p. 500, 1984, p. 295, 1990a, p. 12, 1992, p. 8, and 1995b, p. 259.

5 For a detailed reconstruction of the epistemological part of Quine's global structuralism, see Janssen-Lauret 2019. A critical discussion of this doctrine is to be found in Rosner 1996. 
epistemologically indifferent: there can be no evidence for one ontology as over against another. ${ }^{6}$ On the other hand, there can also be no doubt that his global structuralism is a generalization of the structuralist view in the philosophy of mathematics. The point of the latter is that the truth of a mathematical theory does not depend on the choice of its objects. Mathematical theories 'abstract away' from the nature of objects instantiating mathematical structures. ${ }^{7}$ We can always replace its objects with other objects without changing the truth value of any sentence. There are some places where Quine seems to generalize this semantic claim:

Our overall scientific theory demands of the world only that it be so structured as to assure the sequences of stimulation that our theory gives us to expect. More concrete demands are empty, what with the freedom of proxy functions. (Quine 1981b, p. 22)

This passage strongly suggests that the truth of our overall theory of the world does not depend on the objects we choose for it. But there are also passages in which he seems to hold that the choice of objects does matter to the truth of a theory:

[...] it is a confusion to suppose that we can stand aloof and recognize all the alternative ontologies as true in their several ways, all the envisaged worlds as real. It is a confusion of truth with evidential support. (Quine 1981b, p. 21)

In this case, however, the choice of objects does matter to a scientific theory: its truth depends on this choice. This is a central thesis of Quine's theory of ontological commitment.

Now, Quine's recently published Kant Lectures from 1980 throw new light on his global structuralism. He explicitly says that the reformulation of a theory by means of proxy functions preserves the truth values of all sentences:

Thus imagine any specifiable correlation $\mathrm{f}$ between bodies and any objects you like. Perhaps the latter objects are bodies in turn, so that the correlation is a mere permutation, or perhaps they are things of some other sort. Now anything we care to say about bodies, however lofty or mundane, can be rephrased so as to treat rather of the new objects, simply by incorporating ' $f$ ', the proxy function as I call it, into all the terms. Thus instead of saying of a body that it is a $\mathrm{P}$, we say of the corresponding new thing that it is the $\mathrm{f}$ of a P. We do not actually write this; we just adhere to the old term ' $\mathrm{P}$ ' but reinterpret it as ' $\mathrm{f}$ of a $\mathrm{P}$ '. Everything remains true that was true before, right down to observation sentences.

The same point emerges from Tarski's definition of truth. His definition proceeds by a recursive definition of satisfaction over the ontology of the object language, but any other

6 See Quine 1992, p. 8.

7 See, for instance, Reck 2019, p. 1. 
ontology in specifiable correlation with that one will produce the same result, the sane [sic] truth class, if we merely adapt all terms in the way just described. (Quine 2019, p. 82)

The point of Tarski's definition of truth to which Quine alludes is precisely that we can replace the extensions that a Tarskian definition of truth assigns to the terms of a language by other isomorphic extensions without changing the truth value of any sentence. This is famously proved by Frege's permutation argument (Frege 1893, § 10). Let $\mathrm{f}$ be a proxy function mapping the numbers one-to-one to any objects as proxies such that not every number is mapped to itself. Its value for the number 5 as argument may be Julius Caesar, for instance. Then the proxies can be assigned to the terms of arithmetic as extensions without changing the truth value of any sentence of arithmetic. Since $\mathrm{f}$ is one-to-one, two numbers are identical if and only if their proxies are identical. Hence, the truth value of Hume's principle

(HP) The number of $F s$ = the number of $G s$ iff the $F$ s and the $G$ s can be correlated one-to-one

does not change when we reinterpret the singular terms of arithmetic by means of the proxy function $\mathrm{f}$. The point of this argument is that it justifies the ontological reduction of numbers to their proxies. It establishes that (HP) is ontologically indifferent in the sense that it does not matter to its truth which ontology we choose for it. We are free to identify the number 5 even with Julius Caesar.

Quine's remarks on the Caesar problem in 'Naturalism; or Living Within One's Means' (1995) clearly show that his proxy-function argument is meant as a generalization of Frege's permutation argument:

[...] think of a body in the scientific framework of space and time. Insofar as you specify the precise sinuous filament of four-dimensional space-time that the body takes up in his career, you have fixed the object uniquely. We could go farther and identify the object, a chipmunk perhaps, with its portion of space-time [...]. Thus we can come to terms somewhat with the indeterminacy of reference, as applied to bodies and other sensible substance, by just letting the sensory connotations of the observation sentences carry over from the old objects to their proxies. In the case of abstract objects such as numbers, devoid of sensory connotations, the indeterminacy of reference is already familiar. It is seen in Frege's so-called Caesar problem: the number 5 may be Julius Caesar. We happily use numbers without caring whether they be taken according to the Frege-Russell constructions or Ackermann's or von Neumann's. (Quine 1995a, p. 259)

According to the new interpretation I wish to propose, Quine's thesis of the ontological indifference of theories is basically a semantic thesis claiming that the original ontology of a theory can always be reduced to alternative ones. Ontological indifference is multiple ontological reducibility. An ontological reduction in the relevant sense is a rule for translating the original formulation of a theory 
into an alternative formulation such that the truth of the alternative formulation does not presuppose the existence of all entities whose existence is presupposed by the truth of the original formulation. The thesis of ontological indifference accordingly claims that the truth of a theory does not depend on the existence of its original objects, because it can always be reformulated in such a way that its truth depends only on the existence of some other objects.

This semantic reading is also strongly suggested by the fact that Quine derives his global structuralism from the principle of the semantic primacy of sentences: ${ }^{8}$

Structure is what matters to a theory, and not the choice of its objects. F.P. Ramsey urged this point fifty years ago, arguing along other lines, and in a vague way it had been a persistent theme also in Russell's Analysis of Matter. But Ramsey and Russell were talking only of what they called theoretical objects, as opposed to observable objects. I extend the doctrine to objects generally, for I see all objects as theoretical. This is a consequence of taking seriously the insight that I traced back from Bentham-namely, the semantic primacy of sentences. (Quine 1981b, p. 20)

The present paper aims to reconstruct the version of Quine's global structuralism that results from taking seriously the semantic primacy of sentences. In Section 1, the semantic reading of the thesis of the ontological indifference of theories is explained more closely. In the remaining Sections 2 to 4, its derivation from the principle of the semantic primacy of sentences is reconstructed. Three versions of this principle are discussed. The first is a context principle according to which words have meaning only in the context of a sentence. It is used by Quine to paraphrase away certain entia non grata such as Pegasus and impure numbers. The second version is the behaviorist principle that the reference of words is determined solely by the correlation of sentences with sensory stimulations in our verbal behavior. It is used by Quine to establish the referential indeterminacy of theoretical terms. Finally, the third version is Ramsey's principle that the theoretical terms of a theory are implicitly defined by the Ramsey sentence of that theory. It is implicitly used by Quine in the Kant Lectures to illustrate the ontological indifference of theories. I argue that neither of these principles justifies the reinterpretation of terms that Quine uses to establish the thesis of ontological indifference. The main problem is that his ontological reductions do not account for some hidden ontological presuppositions that are not detected by his criterion of ontological commitment.

8 See also the parallel comments in the Kant Lectures (Quine 2019, p. 82). 


\section{The Content of the Thesis of Ontological Indifference}

According to the Platonic point of view in the philosophy of mathematics, the number 5 is a unique abstract object. A materially adequate definition of this number must accordingly specify which object is denoted by 'the number 5', that is, it must decide for any object $x$ whether ' $x=$ the number 5 ' is true or false. This assumption is denied by the structuralist approach in mathematics. Its principal claim is that mathematical objects are exhaustively defined by their place in the relevant structures. To be the number 5 is to be the sixth member in a progression. Relative to the progressions in which Frege's number 5 is the sixth member, Frege's number 5 is the number 5 , and relative to the progressions in which Julius Caesar is the sixth member, he is the number 5 . Taken by itself, no object is the number 5 . We must hence stipulate, by means of a definition, which object the number 5 is supposed to be. ${ }^{9}$ According to Frege's definition, the number 5 is the set of all sets with 5 elements, while, according to von Neumann's definition, the number 5 is the set of the predecessors of 5 . Both definitions are perfectly adequate, though they are incompatible with each other. This is the multiple-reduction argument that Benacerraf used in his influential critique of mathematical Platonism. ${ }^{10}$

The structuralist thesis that mathematical objects are exhaustively defined by their place in mathematical structures can be understood both as an ontological and a conceptual thesis. Understood as an ontological thesis, it claims that there are no facts deciding which object the number 5 is, whether it is the set of all sets with 5 members or Julius Caesar, for instance. Mathematical objects have only structural properties. When we consider global structuralism as a generalization of this thesis, it claims that there are also no facts determining which object the dog Fido really is. No object is Fido by itself. We must hence stipulate which object Fido is supposed to be. Relative to one definition, Fido is the material content of a certain space-time region, while, relative to another definition, he is a certain pure set.

It is widely assumed that Quine's thesis of ontological relativity involves a generalization of the structuralist ontology of mathematical objects according to which also physical objects have a purely structural nature. On this reading,

\footnotetext{
9 There are structuralist approaches in mathematics according to which numbers are not objects at all, but 'positions in a structure'. They are irrelevant for our discussion here. For an overview over the structuralist approaches in the ontology of mathematics, see Gasser 2015.

10 The locus classicus of this critique is Benacerraf 1965. Quine (1992, p. 5) uses the same argument to defend his structuralist ontology of mathematical objects.
} 
however, the thesis of ontological relativity undermines Quine's realism about physical objects (Quine 1981b, p. 2). If his realism allows us to consider physical objects as pure sets, it does not really claim that there are physical objects. To resolve this tension, Peter Hylton assumes that Quine's realism about physical objects does not refer, in the first place, to a certain kind of objects, but to the role that these objects are supposed to play in theories. To be a realist about physical objects in Quine's sense means to take their role in theories seriously. ${ }^{11}$ In other words: a Quinean realist about physical objects is a structuralist who holds that there is at least one kind of object that is suitable to play the role of physical objects, whatever those objects may be.

However, according to Quine's official explication of his notion of physical object, to be a physical object is to be the material content of a space-time region (see Quine 1981b, pp. 10-13). Physical objects are not defined by their place in structures, but by their material nature. Moreover, physical objects enjoy a privileged status in Quine's ontology. He assumes that the 'conceptual scheme' of physical objects is not acquired by learning the meaning of physical terms like 'dog', but it derives from an innate predisposition to divide the world into physical bodies like dogs that he calls our 'bodymindedness'. We are born as realists about physical bodies. They are our paradigm for what counts as real. ${ }^{12}$ Physical objects are accordingly welcomed with open arms by Quine in his ontology. Sets, by contrast, are only grudgingly acknowledged by him because they are indispensable in science (Quine 1981b, pp. 13-14).

For these reasons, we should not ascribe to Quine a structuralist ontology of physical objects, but a realist one. In 'Relativism and Absolutism' (1984, p. 295), he makes it clear that the proxy-function argument does not undermine his 'robust realism', which is part and parcel of his naturalism, and, in 'Structure and Nature' (1992, p. 9), he explicitly says that his global structuralism 'should not be seen as a structuralist ontology'. ${ }^{13}$

Understood as a conceptual thesis, the structuralist claim that mathematical objects are exhaustively defined by their place in mathematical structures does not refer to mathematical objects, but to mathematical notions. Our notion of the number 5, for instance, is exhaustively captured and fully explained by the notion of being the sixth element in a progression. To be the number 5 means to have this position in such a structure. This is a semantic thesis about the meaning of mathematical terms, and not an ontological thesis about the nature of

11 See Hylton 2007, pp. 317-323, 2004, pp. 144-145, and 2000, pp. 298-299.

12 See Quine 1973, §§ 14, 23, 34, 1981b, p. 2, and 1995a, p. 40.

13 The same clarification is made by him already in 'Things and Their Place in Theories' (1981b, p. 21). 
mathematical entities. It does not rule out that we identify the number 5 with a specific object, say, the set of all sets with exactly five elements. In this case, we have two notions of number, a general notion and a specific one. The general notion is defined in purely structural terms, and the specific notion is defined in terms of a specific class of objects that are supposed to instantiate this structure.

In the Kant Lectures, Quine illustrates his structuralism with Ramsey's method of defining theoretical terms. ${ }^{14}$ The observational terms of a theory $\mathrm{T}$ are regarded as categorematic terms whose semantic interpretation is already fixed. The theoretical terms, by contrast, are treated as syncategorematic terms that are implicitly defined by the Ramsey sentence of $T$. Quine does not explain Ramsey's method more closely. It can be roughly described as follows. ${ }^{15}$ Let $C$ be the conjunction of the axioms of T. $C$ is a single sentence that can be regarded as a reformulation of $T$. Let $C\left[x_{1}, \ldots, x_{\mathrm{n}}\right]$ be the open sentence that results from replacing all theoretical terms $t_{1}, \ldots, t_{\mathrm{n}}$ that occur in $C$ by the variables $x_{1}, \ldots, x_{\mathrm{n}}$, respectively. Call an n-tuple satisfying the open sentence $C\left[x_{1}, \ldots, x_{\mathrm{n}}\right]$ under the intended interpretation of all observational terms a 'realization' of $T{ }^{16}$ The Ramsey sentence of $T$ is the existential sentence $\exists x_{1}, \ldots, \exists x_{\mathrm{n}} C\left[x_{1}, \ldots, x_{\mathrm{n}}\right]$. It claims that $T$ has at least one realization.

In contrast to the original formulation of $T$, the Ramsey sentence of $T$ does not specify what the objects of $T$ are. It 'abstracts away' from the nature of the objects instantiating the empirical structure described by $T$. In the Kant Lectures, Quine calls this Ramsey's 'anonymization' of the objects of a theory. It reveals the structuralist character of all theoretical notions, including the physical ones:

This structuralistic character not only of class talk but of theoretical terms generally must have been appreciated long since and many times. In a vague way it was a recurrent theme in Russell's Analysis of Matter. Ramsey's anonymization is an effective way of driving it home. It is already implicit in the recognition of sentences as primary in semantics: in the recognition that words depend for their meaning on sentences. This insight is traceable to Jeremy Bentham's theory of fictions. (Quine 2019, p. 82)

In the philosophy of mind, Ramsey's method is used to define mental states in such a way that they can be ontologically realized in multiple ways. It can be used to define physical objects in the same way. The Ramseyfied notion of Fido leaves open whether Fido is ontologically realized by the material content of the spacetime region occupied by him or by pure sets. To be Fido is no more or less than to

\footnotetext{
14 A similar illustration is to be found in 'Structure and Nature' (1992, p. 5).

15 Cf. the classical presentation of this approach in David Lewis 1970.

16 This is the terminology used in Lewis (1970, p. 430).
} 
occupy a certain place in the empirical structure of our overall theory of the world. Any object satisfying this role can be regarded as Fido.

To take this into account, we must assume that Quine has two notions of physical object: the Ramseyfied notion, which leaves open how these objects are ontologically realized, and the realist notion, according to which physical objects are the material contents of the space-time regions they occupy. Quine uses the Ramseyfied notion to explain the semantics of physical terms. It corresponds to the general notion of number in mathematical structuralism. The realist notion is used by him to specify which objects there are. It belongs to his ontology.

The Ramsey sentence of our overall theory of the world is true not only in a world whose ground elements are physical objects, but also in a world that consists exclusively of pure sets. It is not committed to any specific ontology. ${ }^{17}$ It demands of the world only that it be so structured as to assure the sequences of stimulation it gives us to expect. Ramsey's anonymization makes thus the ontological indifference of theories explicit: the choice of objects does not matter to their truth. According to this approach, an ontological reduction is materially adequate already if it preserves the structure of the original objects of the theory. ${ }^{18}$ Let $<o_{1}$, $\ldots, o_{\mathrm{n}}>$ be the original objects realizing a theory $T$. A reduction of these objects by means of a proxy function $\mathrm{f}$ is materially adequate iff $\left\langle f\left(o_{1}\right), \ldots, f\left(o_{\mathrm{n}}\right)\right\rangle$ is also a realization of $T$. Prima facie, empirical theories can be realized only by empirical objects, and not also by abstract ones. The reason is that abstract objects are not observable; they are not connected to the empirical data at all. The epistemological point of Quine's global structuralism is that this contrast is an illusion. Physics can be realized by pure sets. ${ }^{19}$

In recent years, Frederique Janssen-Lauret has published some very elucidating papers about the historical development of Quine's global structuralism and its place in his overall philosophical system. ${ }^{20}$ According to her reconstruction, Quine derives his global structuralism from the doctrine of his naturalized epistemology that the conceptual scheme of physical objects is a human invention whose task is to link sensory stimulation to sensory stimulation in a lawlike manner. We know Fido only in virtue of the patterns of stimulation that are caused by him at our sensory receptors. We recognize him as the same object again only in virtue of the place he occupies in the empirical structure of our observations. But we lack identifying knowledge about Fido. There are other objects linking sensory

17 The same observation is made in Janssen-Lauret and MacBride 2020, p. 22. I am grateful to an anonymous reviewer for bringing this to my attention.

18 In Quine's words: 'Save the structure and you save all' (Quine 1992, p. 8).

19 See Quine 2019, p. 82, and also Quine 1981b, p. 16.

20 See Janssen-Lauret 2015, 2016, 2017, 2019, and Janssen-Lauret and MacBride 2020. 
stimulations to sensory stimulations in exactly the same way. They can take on the role that Fido plays in the systematic linking of sensory stimulation to sensory stimulation. Consequently, our knowledge of Fido leaves open which object he is. By the same token, our theories leave open what their objects are. They do not determine the reference of our terms in a 'singular' way. The ontological indifference of theories is thus a consequence of our incomplete knowledge of their objects. ${ }^{21}$

I agree with Janssen-Lauret that Quine did not adopt a deflationist conception of ontology in his mature period. ${ }^{22}$ In contrast to Carnap, he did not defend the view that the choice of objects for a theory is merely a practical question without any theoretical consequences. ${ }^{23}$ But, in my view, there is an important tension between Quine's structuralism and his substantialist conception of ontology that JanssenLauret largely ignores. According to Quine's criterion of ontological commitment, the truth of a sentence depends on the objects we choose as values for the bound variables. It implies that the choice of objects does matter to a theory. Assume, for instance, that only concrete objects like Fido exist, and not also abstract objects like the space-time region of Fido. In this case, the paraphrase or reinterpretation of 'There are dogs' as 'There are space-time regions of dogs' does not preserve the truth value of the original sentence. Consequently, we cannot ascribe to Quine the radical view that the choice of objects does not matter to theories at all. Let $T_{1}$ and $T_{2}$ be two empirically equivalent systems of the world with different ontologies. According to $T_{1}$, the world consists exclusively of pure sets, whereas, according to $T_{2}$, the world contains also physical urelements. Quine explicitly rejects the claim that the hyper-Pythagorean ontology of $T_{1}$ and the physicalist ontology of $T_{2}$ are both true. His argument is that we must not confuse truth with evidential support.

On the other, Quine also explicitly says that $T_{1}$ can be considered as a reformulation of $T_{2}$ that preserves the truth values of all sentences of $T_{2}$. This seems to imply that the choice of objects does not matter to the truth of a theory. How are we to resolve this conflict? As far as I can see, Jannsen-Lauret does not answer this question. ${ }^{24}$ She does not consider Quine's structuralism primarily as a thesis about the truth of theories. My answer is that we must understand the independence of the truth of a theory from the choice of its objects in terms of ontological reducibility. $T_{2}$ does presuppose the existence of physical objects. But this presupposition is not essential to $T_{2}$, because $T_{2}$ can be reduced to $T_{1}$. The point of the ontological reductions is to show that we can free $T_{2}$ from its commitment to

21 See especially Janssen-Lauret and MacBride 2020, Section 2.

22 See Janssen-Lauret 2015, pp. 153-154, and 2017, pp. 249-254.

23 Cf. Carnap 1952.

24 See Janssen-Lauret and MacBride 2020, Section 3, especially p. 11. 
physical objects without changing $T_{2}$ in any substantial way. It is in this sense that the choice of objects does not matter to the truth of a theory.

In what follows, I shall assume that the thesis of the ontological indifference of a theory is a semantic thesis claiming that the ontology of a theory is always reducible to various alternative ontologies. The task of the proxy-function argument is to show how the ontology of our overall theory of the world can effectively be reduced to an alternative ontology. Quine's method of ontological reduction is the contextual definition of theoretical terms. It is based on the principle that sentences are the primary units of semantics, which I shall henceforth call the 'context principle'. Although the choice of objects does not matter to a scientific theory, it still matters to ontology, in Quine's view. Its task is to specify which sorts of objects actually realize our overall theory of the world. To answer this question, he uses his realist notion of physical objects.

\section{The Principle that Words Have Meaning Only in the Context of a Sentence}

Quine's theory of ontological commitment is a semantic theory of the ontological truth conditions (or 'presuppositions') of sentences. Its task is to specify which ontological demands the world must satisfy to make a given sentence or class of sentences true.

To determine the ontological presuppositions of a sentence, we must determine which expressions occurring in the sentence are referential expressions and which are syncategorematic ones. As Quine observes, there are occurrences of proper names in true sentences that are not referential. His most famous example is the occurrence of 'Pegasus' in 'Pegasus does not exist'. If this occurrence were referential, the truth of 'Pegasus does not exist' would presuppose the existence of Pegasus. But, in this case, the sentence would have contradicting truth conditions.

To show that 'Pegasus does not exist' can be true, although 'Pegasus' is not a referential term, we must reformulate this sentence in such a way that 'Pegasus' does not occur at a referential position. Quine's solution to this problem is based on a context principle that goes back to Bentham, Frege, and Russell. ${ }^{25}$ It claims that words have meaning only in the context of sentence (Quine 1948, p. 6). To explain the meaning of a word, we must hence explain the meaning of the sentences in which it occurs. This is exactly what a contextual definition (or 'paraphrase') does.

25 See Quine 1981b, pp. 68-70. 
A paradigmatic example is Russell's contextual definition of the definite description 'the present King of France': ${ }^{26}$

(K) The present King of France is bald iff there is one and only one $x$ that is presently a king of France and that $x$ is bald.

On the categorematic reading of 'the present King of France', the definiendum 'The present King of France is bald' says about the present King of France that he is bald. The task of $(\mathrm{K})$ is to reformulate this sentence in such a way that it does not refer to this non-existent object. The definiens 'There is one and only one $x$ that is presently a king of France and that $x$ is bald' contains a single categorematic expression, the bound variable ' $x$ '. It refers to all existent objects. This sentence says about all existent objects that there is one among them that is presently a king of France and bald and that no other object is presently a king of France.

In the same way, we can translate 'Pegasus does not exist' into a sentence that refers only to existent objects. The contextual definition reads:

(P) Pegasus does not exist iff there is no $x$ such that $x$ is Pegasus.

On the intended reading, the occurrence of 'Pegasus' in the definiens is syncategorematic. To make this explicit, Quine paraphrases ' $x$ is Pegasus' as ' $x$ pegazises' (Quine 1948, p. 8).

He celebrated the discovery of the method of contextual definition as a milestone of philosophy that revolutionized our philosophical inquiries. It was used by Russell to construct a theory of classes that does not presuppose the existence of classes, and by Carnap to translate sentences that refer to physical objects into sentences that refer only to sense data. ${ }^{27}$ Contextual definitions play a fundamental role also in Quine's own philosophy. Their task is to 'paraphrase away' certain entia non grata. Thus, the paraphrase in (P) allows us to deny the existence of Pegasus without presupposing that there are non-existent objects, and the contextual definition of 'The height of Mount Everest $=8849$ m' as 'The height of Mount Everest in meters $=8849$ ' shows how impure numbers can be reduced to pure ones. ${ }^{28}$

Now, in 'Things and Their Place in Theories' (1981), Quine sketched a generalized method of contextual definition that is designed to show that the ontology of

26 See Russell 1905.

27 See Quine 1981c, pp. 68-70, 1981b, p. 3, and 1968b, pp. 72-73.

28 Cf. Quine 1981b, p. 14. Moreover, in Set Theory and Its Logic (1969b, p. 16), Quine uses the contextual definition ' $y \in\{x: \mathrm{F} x\}$ iff Fy' to reformulate a larger part of classical set theory in such a way that the truth of its sentences does not presuppose the existence of sets. 
our overall theory of the world can be reduced to the ontology of pure set theory. It is characterized by the use of proxy functions:

All that is needed [...] is a rule whereby a unique object of the supposedly new sort is assigned
to each of the old objects. I call such a rule a proxy function. Then, instead of predicating a
general term ' $P$ ' of an old object $x$, saying that $x$ is a $P$, we reinterpret $x$ as a new object and say
that it is the fof a $P$, where ' $f$ ' expresses the proxy function. Instead of saying that $x$ is a dog, we
say that $x$ is the lifelong filament of space-time taken up by a dog. Or, really, we just adhere to
the old term ' $P$ ', 'dog', and reinterpret it as ' $f$ of a $P$ ', 'place-time of a dog'. (Quine 1981b, p. 19)

We can explain this method as follows. The function the space-time region of $x$ correlates physical objects one-to-one with the space-time regions they occupy. It can be used to paraphrase (or 'translate')

(1) Fido is a dog

as

(2) The space-time region of Fido is the space-time region of a dog.

On the intended reading, (1) and (2) differ with regard to their ontological presuppositions: whereas (1) presupposes the existence of dogs, (2) presupposes only the existence of space-time regions. ${ }^{29}$ However, (2) contains a hidden quantification over dogs. An object $x$ is the space-time region of a dog only if there is an object $y$ such that $y$ is a dog and $x$ is the space-time region of $y$. Hence, also (2) presupposes the existence of dogs. Quine does not address this problem, presumably because its solution is too obvious. We saw that he paraphrases 'There is no $x$ such that $x$ is Pegasus' as 'There is no $x$ such that $x$ pegasizes' to exclude the referential reading of 'Pegasus' in ' $x$ is Pegasus'. In the same way, we can exclude the referential reading of 'Fido' and 'dog' by paraphrasing (2) as

(3) The fidoizing space-time region is a dogizing space-time region.

Since the singular term 'the fidoizing space-time region' is a definite description, it can be contextually defined by means of Russell's method. The final paraphrase is an existential sentence that can be formulated as follows:

(4) There is one and only one $x$ such that $x$ is a fidoizing space-time region and this $x$ is also a dogizing space-time region.

According to Quine's criterion of ontological commitment, (4) actually presupposes only the existence of space-time regions, and not also of dogs, because

29 Quine (1981b, pp. 17, 19) maintains that an ontology containing both physical objects and space-time regions is 'redundant'. Clearly, the ontological reduction of physical objects to pure sets is supposed to eliminate this kind of redundancy. 
the objects that must be assigned to the bound variable in (4) in order to make this sentence true are only space-time regions, and not also dogs. ${ }^{30}$ The same method can be used to translate (4) into a sentence whose variables refer exclusively to space-time points and ultimately into a sentence whose variables refer exclusively to pure sets.

However, we can show that Quine's ontological reductions are compromised by some hidden ontological presuppositions that his criterion of ontological commitment does not detect. By definition, a space-time region dogizes if and only if it is occupied by a dog, just as an object pegasizes if and only if it is identical to Pegasus. (4) contains a hidden quantification over dogs. Hence, the truth of (4) still presupposes the existence of dogs. (1) and (4) have exactly the same truth conditions. The syncategorematic treatment of expressions affects only the truth conditions of negative existential sentences: while the paraphrase of 'Pegasus exists' as 'There is one (and only one) $x$ that pegasizes' still presupposes the existence of Pegasus, the paraphrase of 'Pegasus does not exist' as 'There is no $x$ such that $x$ pegasizes' does not have this presupposition. Analogously, while (4) still presupposes the existence of Fido, the (sentential) negation of (4) does not presuppose his existence.

The hidden ontological presupposition of (4) is not detected by Quine's criterion because it does not register the ontological presuppositions of hidden quantifications. Let Paul be a married man. According to Quine's criterion, the truth of 'Someone is married' presupposes only the existence of at least one object that satisfies the open sentence ' $x$ is married'. Hence, the existence of Paul is already sufficient to account for the truth of the sentence. However, the predicate ' $x$ is married' is an abbreviation of the predicate ' $x$ is married to someone', which has the logical structure of 'there is a person $y$ and $x$ is married to $y$ '. Consequently, the sentence 'Someone is married' can be true only if there are at least two persons $x$ and $y$ such that $x$ is married to $y$. It can be true in a domain whose sole element is Paul only if outside the domain there is a second person to whom Paul is married. Hence, the sentence 'Someone is married' demands of the world that there be at least two persons. ${ }^{31}$ To assess its ontological presuppositions correctly, we must take the hidden ontological presuppositions into account.

For analogous reasons, (4) can be true in a domain consisting exclusively of space-time regions only under the condition that there are dogs outside the

30 The classical formulation of the criterion reads: 'A theory is committed to those and only those entities to which the bound variables of the theory must be capable of referring in order that the affirmations made in the theory be true' (Quine 1948, pp. 13-14).

31 A similar criticism is made in Rayo 2007, pp. 431-432. For a more thorough discussion of this problem, see Bricker 2014, Section 1.7.4. 
domain. In a world in which dogs do not exist there are no dogizing space-time regions. Hence, Quine's conclusion that '[o] ur overall scientific theory demands of the world only that it be so structured as to assure the sequences of stimulation that our theory gives us to expect' (Quine 1981b, p. 22) cannot be derived from the proxyfunction argument. The contextual definitions do not really allow us to change the ontological presuppositions of our theory in the relevant way.

This does not mean that there are no contextual definitions that allow us to make successful ontological reductions. Consider Frege's contextual definition of the number 1 by

(5) The number of $F \mathrm{~s}=1$ iff there is one and only one $F$, which is a part of his inductive definition of number in Grundlagen (1884). ${ }^{32}$ The definiens of (5) does not presuppose the existence of the number 1. It can be true in a world in which numbers do not exist. Consequently, (5) achieves a successful ontological reduction of the number 1. But the same does not apply to the contextual definition of (1) by (4): the truth of (4) still presupposes the existence of Fido.

Note also that the notion of ontological commitment is an extensional notion, and not an intensional one. Assume that Fido is the dog owned by Peter. In this case,

(6) The dog owned by Peter is a dog

also presupposes the existence of Fido. There are possible worlds in which (6) does not presuppose the existence of Fido, but only the existence of Peter. But, in the actual world, (6) is true only if Fido exists. Hence, the paraphrase of (1) as (6) does not eliminate the ontological presupposition of (1) that Fido exists. ${ }^{33}$

\section{The Behaviorist Context Principle}

In our first reconstruction, we have used a weak version of the context principle according to which all words except the variables are syncategorematic expressions. Our semantic framework was the standard model-theoretical interpretation of first-order logic, which goes back to Tarski. It is characterized by the assignment of a domain to the bound variables. The theory of ontological commitment in 'On What There is' (1948) is embedded in this semantic framework.

32 See Frege 1884, § 55. I am simplifying his definition here.

33 I am grateful to an anonymous reviewer for suggesting (6) as a counter-example to my claim that the paraphrase of (1) as (4) does not free us from the ontological commitment to Fido. For a comparison of Quine's extensional criterion of ontological commitment with intensional ones, see Bricker 2014, Sections 1.4 and 1.5, and also Rayo 2007, Section 1. 
To this it might be objected that the theory of ontological reduction in 'Things and Their Place in Theories' (1981) is based on a behaviorist (or verificationist) theory of language. This is suggested by the following remarks on the semantic primacy of language:

This [i.e. global structuralism] is a consequence of taking seriously the insight that I traced back from Bentham-namely, the semantic primacy of sentences. It is occasion sentences, not terms, that are to be seen as conditioned to stimulations. Even our primordial objects, bodies, are already theoretical - [...]. It is occasion sentences that report the observations on which science rests. The scientific output is likewise sentential: true sentences, we hope, truths about nature. The objects, or values of variables, serve merely as indices along the way, and we may permute or supplant them as we please as long as the sentence-to-sentence structure is preserved. The scientific system, ontology and all, is a conceptual bridge of our own making, linking sensory stimulations to sensory stimulations. (Quine 1981b, p. 20)

According to Quine's behaviorist theory of language, the reference of linguistic expressions is determined exclusively by our publicly observable linguistic behavior in publicly observable circumstances. Our mental states are irrelevant. ${ }^{34}$ The linguistic behavior connecting language to the world consists exclusively in our dispositions to accept a given sentence as true or to reject is as false when our sensory receptors are triggered by suitable patterns of stimulations. The patterns prompting assent to a sentence are its 'positive stimulus meaning', and the patterns prompting dissent are its 'negative stimulus meaning'. The stimulus meaning of a sentence is the ordered pair consisting of its positive and its negative stimulus meaning (Quine 1968b, p. 81).

According to this approach, the observation sentence 'This is a dog' is connected to the world only holophrastically, in virtue of its stimulus meaning, and not also compositionally, in virtue of its Tarskian satisfaction conditions. ${ }^{35}$ Its truth in a given context of utterance does not presuppose the presence of a dog, but only the presentation of a pattern of stimulation that belongs to the positive stimulus meaning of this sentence (Quine 1992, p. 29). Because of this feature, observation sentences are ontologically neutral; they do not have any ontological presuppositions.

According to the behaviorist context principle, words are connected to the world only in an indirect way, in virtue of the correlation of sentences with patterns of stimulation. ${ }^{36}$ The only means we have to fix the reference of 'dog' are observable gestures such as the pointing to a dog, accompanied with an utterance of the sentence 'This is a dog'. This amounts to the ostensive definition of 'dog' in terms of the sentence 'This is a dog'. It is this sentence, and not the term 'dog', that is to be

34 See Quine 1968a, pp. 27-28.

35 Compare, for instance, Quine 1992, p. 8 and 1968a, p. 27.

36 See, for instance, Quine 1960, p. 35. 
seen as conditioned to stimulations. For this reason, sentences are to be seen as the basic units of semantics, in Quine's view.

However, the ostensive definition of a term does not well-define its reference. ${ }^{37}$ Whenever we point to a dog, we simultaneously point to the space-time region occupied by a dog and also to the space-time points of which these regions consist. Consequently, we can reinterpret 'dog' in various ways without changing the stimulus meaning of our sentences. From this follows the epistemic thesis of Quine's global structuralism that the choice of objects does not matter to the connection of a theory with observations:

The apparent change is twofold and sweeping. The original objects have been supplanted and the general terms reinterpreted. [...] Yet verbal behavior proceeds undisturbed, warranted by the same observations as before and elicited by the same observations. Nothing has really changed. (Quine 1981b, p. 19.)

The semantic claim that the choice of objects does not matter to the truth of a theory seems to follow as well. According to the behaviorist view of language, there is no semantic difference between two speakers when there is no corresponding difference in their verbal behavior. ${ }^{38}$ The semantic facts supervene on the behavioral facts. Let A be a speaker with the conceptual scheme of physical bodies and B a speaker with a hyper-Pythagorean scheme according to which the world divides into pure sets. The proxy-function argument shows that there is no behavioral difference between A and B. From this follows Quine's thesis in 'Ontological Relativity' that there is no difference between the specification of the ontology presupposed by a theory and its reduction to another ontology (Quine 1968a, p. 43). Our linguistic behavior does not determine the domain of the bound variables. Consequently, there are no facts that determine the reference of terms. This is Quine's thesis of the indeterminacy of reference (Quine 1968a, p. 47).

Since there are no semantic differences between the sentences affirmed by A and B, they should have the same truth conditions. In this case, the truth of our theories does not depend on the choice of objects we make, but only the sensory stimulations we receive. It is not clear whether Quine actually accepts this conclusion. He does not clearly distinguish between two fundamentally different theories of the truth conditions of sentences that Davidson calls the 'proximal' and the 'distal' theory (Davidson 1990, pp. 73-78). ${ }^{39}$ The proximal theory is a

37 For a detailed reconstruction of the role of ostension in Quine's theory of translation, see Davidson 2003, especially pp. 288 and 291.

38 See Quine 1960, p. 23.

39 The differences between the two theories are explained in more detail in Greimann 2009, pp. 362-367. 
naturalized version of the verificationist semantics of the Vienna Circle, according to which the truth of a sentence depends only on the observations that speak for or against its truth. ${ }^{40}$ It implies that the truth of 'Fido is a dog' does not depend on the properties of Fido, but only on the properties of our sensory stimulations. An utterance of 'There is a dog in front of me' is true in a context of utterance if and only if the speaker receives a sensory stimulation that belongs to the positive stimulus meaning of this sentence. The existence of a dog in the context of utterance is not a condition of its truth.

Quine uses the proximal theory to answer the question of how we should deal with Barry Stroud's skeptical worry that the world might be completely different from the way our sensory impacts lead us to think of it:

What then does our overall scientific theory really claim regarding the world? Only that it is somehow so structured as to assure the sequences of stimulation that our theory gives us to expect. More concrete demands are indifferent to our scientific theory itself, what with the freedom of proxy functions. Our scientific theory can indeed go wrong, and precisely in the familiar way: through failure of predicted observation. But what if, happily and all unknown to us, we have achieved a theory that is conformable to every possible observation, past and future? In what sense could the world then be said to deviate from what the theory claims? Clearly in none, even if we can somehow make sense of the phrase 'every possible observation'. (Quine 1981d, p. 474)

In this passage, Quine holds that the truth of a theory depends only on the observations that count as evidence for or against its truth. Truth and evidential support are one and the same thing. But, in this case, theories are ontologically neutral. They do not have any ontological presuppositions. We do not need a theory of ontological commitment. The choice of objects for a theory does not matter at all. ${ }^{41}$

According to the 'distal' approach, on the other hand, the truth of theoretical sentences depends on the existence and the properties of public objects like dogs. ${ }^{42}$ The sentence 'There is a dog in front of me' is true in a given context of utterance only if there is a real object in front of the speaker that is really a dog. This 'realist' conception of truth is presupposed by Quine's criterion of ontological

40 In 'Epistemology Naturalized' (1968), Quine explicitly adopts the verificationist account of meaning and truth. He agrees with Peirce that 'the meaning of a sentence turns purely on what would count as evidence for its truth' (Quine 1968b, p. 80). And, in Roots of Reference (1973), he justifies the verificationist approach with his theory of language learning. He argues that to learn a language is 'to learn the meaning of its sentences, and hence to learn what observations to count as evidence for and against it' (Quine 1973, p. 38).

41 Carnap (1952) has defended this deflationist view.

42 It is adopted by Quine to do justice that language is a public and social institution, not a private one. For a collection of quotes that suggest this reading, see Davidson 2003, pp. 290-293. 
commitment. It is based on the standard model-theoretical interpretation of sentences in Tarskian semantics, according to which the truth conditions of sentences depend on the objects that we assign to the bound variables.

To make the realist interpretation of sentences with the thesis of the indeterminacy of reference compatible, Quine assumes that sentences have a full modeltheoretical interpretation only relative to their translation into a 'background language' (Quine 1968a, p. 51). This relativization implies his thesis of 'ontological relativity', according to which the ontology presupposed by a theory is relative to the translation of that theory into a background language (Quine 1968a, pp. 48-51). As long as we translate 'Fido' as 'Fido' and 'dog as 'dog', the sentence (1) Fido is a dog

is true if and only if there is one and only one $x$ such that $x$ is Fido and $x$ is a dog (see Quine 1968a, p. 48). When, on the other hand, we translate 'Fido' as 'the spacetime region of Fido' and 'dog' as 'space-time region of a dog', (1) has the realist truth conditions of

(2) The space-time region of Fido is the space-time region of a dog.

To assess the ontological presuppositions of (1) relative to its translation as (2), we must hence apply the criterion of ontological commitment to (2). Relative to this translation, (1) is true if and only if there is exactly one $x$ such that $x$ is the spacetime region of Fido and $x$ is the space-time region of a dog.

According to Quine's criterion of ontological commitment, (2) can be true in a domain that does not contain dogs. Hence, the translation of (1) as (2) allows us to make the ontological reductions we need to establish global structuralism. However, these reductions are again compromised by the hidden ontological commitments of (2). In a world in which dogs do not exist, no space-time region is a space-time region of a dog. Consequently, relative to the translation of (1) as (2), (1) presupposes not only the existence of space-time regions, but also the existence of dogs. The relativization of reference has no effect on the truth conditions of sentences. It cannot be used to eliminate hidden ontological presuppositions.

\section{The Ramseyan Context Principle}

There are some writings in which Quine suggests that the translation of a term into the metalanguage fixes its reference only in a relative way (see Quine 1990b). The translation of 'Fido' as 'the space-time region of Fido' lays down that both terms have the same reference, but it leaves open to which object 'Fido' refers, because the reference of 'the space-time region of Fido' in the metalanguage is also 
indeterminate. This approach amounts to the Ramseyan interpretation of theoretical terms. The Ramsey sentence of a theory $\mathrm{T}$ claims only that $\mathrm{T}$ has at least one realization that accords with the empirical facts. When we translate 'Fido' into a term of the Ramsey sentence of another theory, we hence do not specify to which object 'Fido' refers, but we claim only that 'Fido' and its translation refer to the same object, whatever this object may be. Reference is not a language-world relation, but a language-language relation.

Under this interpretation, the sentence 'Fido is a dog' can actually be true in a world that consists exclusively of space-time regions or pure sets. Theories are ontologically indifferent because the truth of their Ramsey sentences does not depend on the ontological properties of objects, but only on their empirical properties. The problem of hidden ontological commitments does not occur.

It is not clear whether Quine really accepts the reformulation of theories in terms of their Ramsey sentence. In the Kant Lectures, he uses Ramsey's 'anonymization' of theoretical objects to illustrate the structuralist character of theoretical terms. On the other hand, Ramsey's interpretation of sentences is clearly incompatible with Quine's theory of ontological commitment. We already saw that Ramsey's approach is based on a context principle according to which theoretical terms are syncategorematic expressions whose meaning is implicitly defined by the Ramsey sentences of the theory in which they occur. According to this interpretation, singular terms like 'Fido' are variables that are bound by invisible existential quantifiers, while, according to Quine's interpretation, such terms are definite descriptions. To make both interpretations compatible, we must treat 'Fido' both as a variable and as a definite description. To this end, we must transform the Ramsey sentence of our theory into a definite description of Fido. Ramsey's method of defining of theoretical terms is based on this idea. Let $\mathrm{x}_{1}$ be the variable representing 'Fido' in the Ramsey sentence of our theory T. We can then define Fido explicitly as the first element of the n-tupel that uniquely satisfies $C\left[x_{1}, \ldots, x_{n}\right]:{ }^{43}$

(F) Fido $=$ the $x_{1}$ such that $\exists x_{2}, \ldots, \exists x_{n} C\left[x_{1}, \ldots, x_{n}\right] \& \forall y_{1}, \ldots, \forall y_{n}\left(C\left[y_{1}, \ldots, y_{n}\right]\right) \rightarrow x_{1}=y_{1} \& \ldots \&$ $x_{\mathrm{n}}=y_{\mathrm{n}}$.

Obviously, this definition presupposes that $C\left[x_{1}, \ldots, x_{\mathrm{n}}\right]$ has a unique realization. Only in this case the definite description in (F) refers to a well-defined object. If $C$ $\left[x_{1}, \ldots, x_{\mathrm{n}}\right]$ has more than one realization, almost all sentences containing 'Fido' are either false or neither true nor false. They are false on the syncategorematic (Russellean) reading of the definite description, because the uniqueness condition

43 Cf. Lewis 1970, pp. 429, 437-438. I am simplifying Lewis' account here. 
of the definite description is not fulfilled, and they are neither true nor false on the categorematic (Fregean) reading, because the definite description is an empty name. ${ }^{44}$ But, according to Quine's proxy-function argument, $C\left[x_{1}, \ldots, x_{\mathrm{n}}\right]$ has multiple realizations. Hence, we cannot treat the variable 'Fido' simultaneously as a definite description. Under the Ramseyan interpretation,

(7) There is one and only one $x$ that is Fido and that $x$ is a dog

is false, because there is a plurality of objects satisfying the Ramseyfied notion of Fido. For analogous reasons, we cannot treat the second order variable 'dog' simultaneously as a general term. As a consequence, we must consider 'Fido is a dog' as a second order formula to which Quine's criterion of ontological commitment cannot be applied.

Janssen-Lauret and MacBride seem to assume that Quine identifies the ontological commitments of our overall theory of the world with the ontological commitments of its Ramsey sentence. ${ }^{45}$ But, in this case, Quine would have to replace his original criterion of ontological commitment with a Ramseyan one. In order to make the ontological commitments of a theory $T$ explicit, we must not translate $T$ into his regimented first order language, but into the Ramsey sentence $\exists x_{1}, \ldots, \exists x_{\mathrm{n}} C\left[x_{1}, \ldots, x_{\mathrm{n}}\right]$, which is typically a second order formula. This translation makes it explicit that $\mathrm{T}$ demands of the world only that it be so structured as to assure the sequences of stimulation that $\mathrm{T}$ gives us to expect. The problem of the indeterminacy of reference does not arise, because the bound variables of the Ramsey-sentence always refer to all entities of all realizations of the corresponding category.

Moreover, the Ramseyan approach is also incompatible with Quine's naturalized epistemology. Quine assumes that the conceptual scheme of physical objects is a human artefact that plays an important role in the organization of our experience. ${ }^{46}$ But this does not mean that we must consider physical objects as fictional entities. On the contrary, to enjoy the epistemic benefits of this conceptual scheme, we must assign physical objects to the bound variables of the sentences we affirm. Thereby we commit ourselves to the ontology of physical objects. This is the ontological price we have to pay for the epistemic benefits of positing physical objects. We can revise our conceptual scheme and opt for a hyper-Pythagorean scheme. In this case, we commit ourselves to the hyper-Pythagorean ontology of pure sets. But we cannot stand aloof and do science without adopting any

44 Cf. Lewis (1970, p. 438).

45 See Janssen-Lauret and MacBride 2020, Section 5, especially p. 22.

46 See, for instance, Quine 1981b, p. 20 and 1992, p. 6. 
conceptual scheme. ${ }^{47}$ Hence, truth depends on the ontology we accept. Quine calls this the 'immanence' of truth: ${ }^{48}$

[I]t is a confusion to suppose that we can stand aloof and recognize all the alternative ontologies as true in their several ways, all the envisaged worlds as real. It is a confusion of truth with evidential support. Truth is immanent, and there is no higher. We must speak from within a theory, albeit any of various. (Quine 1981b, p. 21)

Obviously, Ramsey's interpretation of scientific language is incompatible with Quine's assumption that we must assign a specific domain of objects to the bound variables. This assumption presupposes again a realist interpretation of language under which the truth of sentences depends on the domain we choose for the bound variables. According to Ramsey's interpretation, we do not choose a particular domain for the bound variables. The variables range over all objects of all domains. Truth is not immanent in the relevant sense.

For the same reasons, Ramsey's interpretation also undermines Quine's theory of ontological reduction. It implies that we cannot reformulate a theory in such a way that the original domain is replaced by an alternative one. The contextual definitions do really not eliminate any ontological presuppositions. Neither the original formulation nor any reformulation presupposes a particular domain. To reduce the original domain of a theory to an alternative one, we again need a realist interpretation that assigns a particular domain to the bound variables.

The conclusion to be drawn is that Quine's global structuralism cannot be derived from any version of the context principle that is suggested in his texts. They do not allow us to make the ontological reductions we need to establish this doctrine. This does not imply that the reductions cannot be justified at all. In 'Whither Physical objects?' (1976), Quine has convincingly shown that the ontological reduction of physical objects to pure sets is suggested by physics itself, because the traditional conception of physical objects as material entities conflicts with some physical phenomena like the lack of identity criteria for electrons over time ${ }^{49}$ There are thus alternative ways to justify his ontological reductions. We can only conclude that these reductions cannot be justified by the context principle.

Research funding: This research was funded by Conselho Nacional de Desenvolvimento Científico e Tecnológico.

47 See also Quine 1995a, p. 9.

48 See also Quine 1984, p. 295, and 1992, p. 9. For a detailed reconstruction of the role of naturalism in Quine's ontology, see Gregory 2019 and Janssen-Lauret 2019.

49 See Quine 1976, pp. 498, 499, 502, 503. This result is considered by him as 'triumph of hyperPythagoreanism'. 


\section{References}

Benacerraf, P. 1965. “What Numbers Could Not Be.” Philosophical Review 74: 47-73.

Bricker, P. 2014. “Ontological Commitment.” In Stanford Encyclopaedia of Philosophy, edited by E. Zalta, https://plato.stanford.edu/entries/ontological-commitment/.

Carnap, R. 1952. "Empiricism, Semantics and Ontology." Reprinted in: Carnap, In Meaning and Necessity. A Study in Semantics and Modal Logic. 2nd ed., 1956, 205-21. Chicago: Chicago University Press.

Davidson, D. 1990. “Meaning, Truth and Evidence." In Perspectives on Quine, edited by

R. B. Barrett, and R. F. Gibson, 68-79. Oxford: Blackwell.

Davidson, D. 2003. “Quines Externalism.” Grazer Philosophische Studien 66: 281-97.

Frege, G. 1884. Die Grundlagen der Arithmetik. Eine logisch mathematische Untersuchung über den Begriff der Zahl, Breslau, 1884. Translated as The Foundations of Arithmetic. A LogicoMathematical Enquiry into the Concept of Number. 2nd ed., 1953. Oxford: Blackwell.

Frege, G. 1893. Grundgesetze der Arithmetik, Vol. I. Jena: Hermann Pohle.

Gasser, M. 2015. "Structuralism and its Ontology." Ergo 2: 1-26.

Gregory, P. 2019. "Quine's Ding an Sich: Proxies, Structure, and Naturalism." In Sinclair 2019, 137-56. Cham: Palgrave Macmillan.

Greimann, D. 2009. “Contextual Definition and Ontological Reduction.” Australasian Journal of Philosophy 87: 357-73.

Hylton, P. 2000. “Reference, Ontological Relativity, and Realism.” Aristotelian Society Supplementary 74: 281-99.

Hylton, P. 2004. “Quine on Reference and Ontology.” In The Cambridge Companion to Quine, edited by R. F. Gibson, 115-50. Cambridge: Cambridge University Press.

Hylton, P. 2007. Quine. London and New York: Routledge.

Janssen-Lauret, F. 2015. “Meta-Ontology, Naturalism, and the Quine-Barcan Marcus Debate.” In Quine and His Place in History, edited by G. Kemp, and F. Janssen-Lauret, 146-67. Cham: Palgrave Macmillan.

Janssen-Lauret, F. 2016. “Committing to an Individual: Ontological Commitment, Reference and Epistemology." Synthese 193: 583-604.

Janssen-Lauret, F. 2017. “The Quinean Roots of Lewis's Humeanism.” The Monist 100: 249-65. Janssen-Lauret, F. 2019. “Quine, Ontology, and Physicalism.” In R. Sinclair 2019, 181-204.

Janssen-Lauret, F., and MacBride, F. (2020). "W.V. Quine and David Lewis: Structural (Epistemological) Humility.” In Quine: Structure and Ontology, edited by F. Janssen-Lauret, 27-55. Oxford: Oxford University Press.

Lewis, D. 1970. "How to Define Theoretical Terms." The Journal of Philosophy 67: 427-46.

Quine, W. V. 0. 1948. “On What There Is.” Reprinted in. Quine 1961: 1-19.

Quine, W. V. 0. 1960. Word and Object. New edition 2013. Cambridge: M.I.T. Press.

Quine, W. V. O. 1961. From a Logical Point of View. 2nd ed. Cambridge: Harvard University Press.

Quine, W. V. 0. 1964. "Ontological Reductions and the World of Numbers." In Quine, the Ways of Paradox and Other Essays. Revised ed., 1979, 212-20. Cambridge: Harvard University Press.

Quine, W. V. O. 1968a. “Ontological Relativity.” Reprinted in: Quine 1969a, 26-68.

Quine, W. V. 0. 1968b. “Epistemology Naturalized.” Reprinted in: Quine 1969a, 69-90.

Quine, W. V. 0. 1969a. Ontological Relativity and Other Essays. New York: Columbia University Press. 
Quine, W. V. 0. 1969b. Set Theory and its Logic. 2nd and Revised ed. Cambridge: Harvard University Press.

Quine, W. V. O. 1973. The Roots of Reference. La Salle: Open Court.

Quine, W. V. O. 1976. "Whither Physical Objects?" In Essays in Memory of Imre Lakatos, edited by R. S. Cohen, P. K. Feyerabend, and M. W. Wartofsky, 487-504. Dordrecht: Reidel.

Quine, W. V. 0. 1981a. Theories and Things. Cambridge: Harvard University Press.

Quine, W. V. O. 1981b. "Things and Their Place in Theories.” In Quine 1981, 1-23.

Quine, W. V. 0. 1981c. "Five Milestones of Empiricism." In Quine 1981, 67-72.

Quine, W. V. O. 1981d. "Reply to Stroud." Midwest Studies in Philosophy 6: 473-75.

Quine, W. V. 0. 1983. “Ontology and Ideology Revisited.” The Journal of Philosophy 80: 499-502.

Quine, W. V. 0. 1984. "Relativism and Absolutism." The Monist 67: 293-6.

Quine, W. V. O. 1990a. Pursuit of Truth. Revised ed., 1992. Cambridge: Harvard University Press.

Quine, W. V. O. 1990b. “Three Indeterminacies.” In: Perspectives on Quine, edited by R. B. Barrett, and R. F. Gibson, 1-16. Oxford: Blackwell.

Quine, W. V. O. 1992. "Structure and Nature.” The Journal of Philosophy 89: 5-9.

Quine, W. V. 0. 1995a. From Stimulus to Science. Cambridge: Harvard University Press.

Quine, W. V. 0. 1995b. “Naturalism; or Living Within One's Means.” Dialectica 49: 251-61.

Quine, W. V. 0. 2019. "The 1980 Immanuel Kant Lectures." In Sinclair 2019, 17-87.

Rayo, A. 2007. "Ontological Commitment." Philosophy Compass 2/3: 428-44.

Reck, E. 2019. "Structuralism in the Philosophy of Mathematics." Stanford Encyclopedia of Philosophy, edited by E. Zalta, https://plato.stanford.edu/entries/structuralismmathematics.

Rosner, J. 1996. “Quine’s Global Structuralism.” Dialectica 50: 235-42.

Russell, B. 1905. “On Denoting.” Mind 14: 479-93.

Sinclair, R., ed. 2019. Science and Sensibilia by W. V. Quine. The 1980 Immanuel Kant Lectures. Cham: Palgrave Macmillan. 